\title{
Geometric Optimization of a Large Scale CDPR Operating on a Building Facade
}

\author{
Hussein Hussein ${ }^{1}$, João Cavalcanti Santos ${ }^{1}$ and Marc Gouttefarde ${ }^{1}$
}

\begin{abstract}
This paper deals with the optimization of the geometry of a Cable-Driven Parallel Robot (CDPR) dedicated to large-scale construction applications. Since the maximum cable tension is a critical parameter in the design of the CDPR components, the geometry of the CDPR is optimized by minimizing the lowest maximum cable tension that ensures the validity of wrench-feasibility constraints. The geometric design procedure used in this paper consists of two phases, the CDPR cable connections is selected in the first phase followed by a second phase where the geometric parameters are optimized. The result of this procedure is an original fully-constrained CDPR geometry.
\end{abstract}

\section{INTRODUCTION}

Cable-driven parallel robots (CDPRs) are a special kind of robot in which a mobile platform is connected to winches by the use of cables. Since long cables can be wound on winch drums, CDPRs can have a wide workspace. Several applications taking advantage of this characteristic were proposed. The NIST RoboCrane and the CoGiRo [1], [2] are suspended CDPRs able to displace heavy objects across a large workspace. Thompson and Blackstone introduced in [3] the SkyCam, a movable camera system actuated by cables is meant for the broadcasting of sport events.

Two types of CDPR configurations can be distinguished. On the one hand, a suspended CDPR has all its cable drawing points located above its workspace, similarly to a crane. The cables remain tensed thanks to the weight of the platform. On the other hand, a fully-constrained CDPR can be obtained by positioning some of the cable drawing points in the lower part of the workspace and the others in the upper part. For the CDPR mobile platform to be fully-constrained by the cables, the cables must be arranged to as to obtain wrench-closure [4], [5].

Examples of fully-constrained CDPRs are the FALCON [4] and the IPAnema family [6], [7]. Lafourcade, Llibre and Reboulet proposed in [8] a similar machine to be used in wind tunnels. In [9], a storage retrieval machine based on a fully-constrained CDPR is presented while the one studied in [10] is designed with the purpose of painting and sandblasting large parts.

The context of this work is the development of new strategies for automation and the incorporation of robotic technologies in the construction sector [11]. In this context, this paper discusses the optimization of the geometry of a CDPR intended to the construction and maintenance of

\footnotetext{
${ }^{1}$ Hussein Hussein, João Cavalcanti Santos and Marc Gouttefarde are with LIRMM, Université de Montpellier, CNRS, Montpellier, France, \{hussein.hussein, joao.cavalcanti-santos, marc.gouttefarde\}@lirmm.fr
}

building facades. Tasks such as the installation of curtain walls and the facade cleaning may have their efficiency improved with the use of a mobile platform able to move in front of the building. Obviously, a wide workspace is necessary in order to work on the whole building facade and heavy payload capabilities are required. Furthermore, since the CDPR may be subjected to substantial disturbances, such as wind and possible interactions with the building, superior stiffness is fundamental. These prerequisites justify the consideration of a fully-constrained CDPR.

The optimization of the geometry of a CDPR is composed of several steps. The first choice to be made is the number of cables. Considering the intended mobility of the platform and for symmetry reasons, the studied CDPR mobile platform is driven by 8 cables. Several studies [2], [6], [8] showed that large 6-DOF CDPRs driven by 8 cables have interesting performances. Once the number of cables driving the platform is chosen, the geometry design problem consists in determining (i) the positions of the cable drawing points at the base frame, (ii) the cable attachment points on the mobile platform, and (iii) the cable connections (or arrangement) between these two sets of points. The present paper focuses on these three problems. To this end, the two-phase geometry design methodology in [2] is used. Inspired by [12], the first phase of this methodology consists in testing a very large number of possible cable connections between various base and platform preselected geometries. The second phase aims at refining the result of the first phase using an optimization algorithm.

Studies on the geometry optimization of CDPRs started relatively recently in [12] where several concepts related to the subject were introduced: Cable collisions, the combinatorial problem of cable connections, and workspace analysis. Similarly to this work, most of the following studies on CDPR design were based on a workspace analysis [2], [6], [13]-[20]. Typically, a global performance index is optimized considering the required workspace [2], [13], [14], [16]. The performance measure used in [2] is the maximum acceptable distance between the mobile platform geometric center and its center of mass. A global conditioning index (GCI) is proposed in [13]. In [19], the largest circle delimiting singular-free area is calculated for several positions of the end-effector (considering a gait rehabilitation trajectory). Using this procedure, geometric parameters are optimized in order to enlarge the wrench-closure workspace. Guay et. al introduced in [21] a kinetostatic index which measures the closeness to instability and presented an example of application to CDPRs. Stiffness [22] may also be used as 
a performance measure. Recently, the simultaneous use of stiffness and dexterity was discussed in [23]. In addition, Zanotto et. al present several performance indexes in [24]. These studies present local indexes. During the geometry optimization, the CDPR performances should be evaluated for a (finite) number of platform poses. A global index can be obtained from this set of platform poses. Alternatively, indexes which are intrinsically global may be proposed. Taking the required workspace as constraint, the space occupied by the CDPR (volume of the basis structure) is minimized in [10], [20]. Besides, Rosati et. al [17] showed that adaptive CDPRs may decouple (totally or partially) the dependency of these local indexes on the CDPR mobile platform pose. As a result, a uniform performance index value may be obtained over the robot workspace.

Alternatively, geometry optimization may be based on a specific trajectory (or on a set of trajectories) [25]-[28] instead of on a workspace analysis. The CDPR which performs the trajectory in the shortest time is chosen in [25], [28]. Bryson, Jin and Agrawal propose in [27] the minimization of the cable tensions required to complete a particular motion. As aforementioned, a critical parameter during the mechanical design of a CDPR is the maximum cable tension. The final cost of the robot is closely related to this parameter. Hence, the approach of minimizing the cable tensions is an appealing choice. Nevertheless, a CDPR is generally expected to present good performances across its workspace and not only on specific trajectories.

This paper deals with the geometry optimization of a large fully-constrained CDPR. More specifically, the geometry optimization presented in this paper aims at reducing the required maximum cable tension over the CDPR workspace. Based on a quasi-static analysis and on purposely defined wrench-feasibility constraints, an original explicit formulation of the lowest possible maximum cable tension permitting to satisfy these wrench-feasibility constraints is introduced. This explicit formulation is the first main contribution of this paper. It is used as the objective function of the CDPR geometry optimization problem. Applying this geometry optimization to the case of a large CDPR intended to work on a building facade, an original fully-constrained CDPR geometry is obtained, which is the second main contribution of this paper.

The basic CDPR modeling used in this paper is presented in Section II. The formulation of the lowest maximum cable tensions allowing to satisfy wrench-feasibility constraints is introduced in Section IV. This formulation is based on the wrench-feasibility conditions presented in Section III and IV. The workspace definition and studied CDPR base and platform geometries are presented in Section V. The design criteria are discussed in Section VI. Finally, Section VII presents the two-phase geometry design procedure and discusses the obtained result which consists of an original fullyconstrained CDPR geometry.

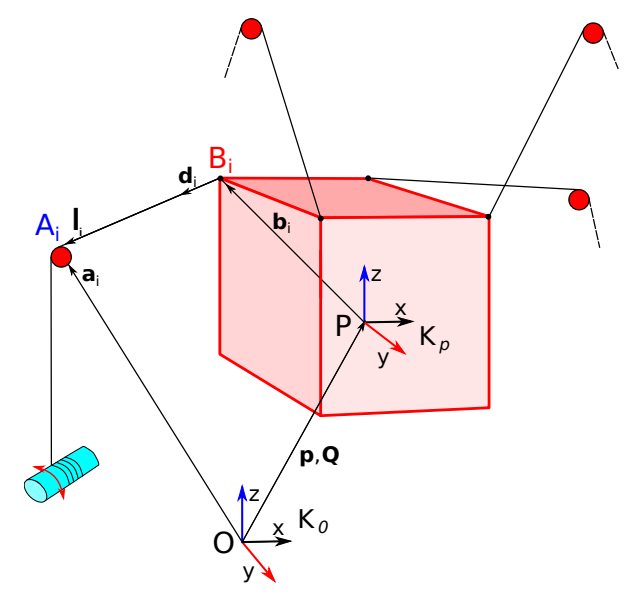

Fig. 1. Schematic representation of the general kinematic parameters of a CDPR.

\section{CABle ROBOt BASIC MOdEling}

A basic CDPR modeling is presented in this section including the notations and the static equilibrium. The dynamics is neglected in the geometry optimization procedure, since the CDPR is expected to work in a quasistatic manner because, for safety purposes, velocities and acceleration should be limited in construction applications. The cable mass, and thus cable sagging, is also neglected. This assumption is reasonable for the design, where a very accurate model is not required contrary to the case of control and calibration.

Figure 1 shows a schematic representation of a CDPR with the notations used in the modeling. The CDPR has six DOFs and its number of cables is denoted by $n$.

The cables are attached to the mobile platform at points $B_{i}$, referred to as the attachment points, and exit from the base at points $A_{i}$, referred to as the drawing points, where $i=1,2, \ldots, n$. Vector $\boldsymbol{a}_{i}$ is the position vector of point $A_{i}$ in $K_{0}(O, x, y, z)$, where $K_{0}$ is a fixed reference frame. Vector $\boldsymbol{b}_{i}$ is the position vector of point $B_{i}$ in $K_{p}(P, x, y, z)$, where $K_{p}$ is frame attached to the CDPR mobile platform, and $P$ is the reference point of the platform which is not necessarily coincident with its center of mass. The position vector of point $P$ in $K_{0}$ is denoted by $\boldsymbol{p}$. The rotation matrix $\boldsymbol{Q}$ defines the orientation of $K_{p}$ with respect to $K_{0}$, i.e., the orientation of the CDPR mobile platform. Vector $\boldsymbol{p}$ and matrix $\boldsymbol{Q}$ define the pose of the mobile platform.

The cable length vector $\overrightarrow{B_{i} A_{i}}$ expressed in the frame $K_{0}$ is denoted by $\boldsymbol{l}_{i}$. Referring to Fig. 1 , it is given by $\boldsymbol{l}_{i}=$ $\boldsymbol{a}_{i}-\boldsymbol{p}-\boldsymbol{Q} \boldsymbol{b}_{i}$. The unit vector $\boldsymbol{d}_{i}$ along $\boldsymbol{l}_{i}$ is equal to $\boldsymbol{d}_{i}=\boldsymbol{l}_{i} / l_{i}$, where $l_{i}$ denotes the length of the straight line segment $B_{i} A_{i}$.

The wrench matrix $\boldsymbol{W}$ provides the relationship between the cable tensions $\boldsymbol{t}$ and the wrench $\boldsymbol{f}$ (forces and moments) applied by the cables on the CDPR mobile platform at point $P$ [14]:

$$
W t=f
$$


The $6 \times n$ wrench matrix $\boldsymbol{W}$ is given by:

$$
\boldsymbol{W}=\left[\begin{array}{cccc}
\boldsymbol{d}_{1} & \boldsymbol{d}_{2} & \cdots & \boldsymbol{d}_{n} \\
\boldsymbol{Q b _ { 1 } \times \boldsymbol { d } _ { 1 }} & \boldsymbol{Q} \boldsymbol{b}_{2} \times \boldsymbol{d}_{2} & \cdots & \boldsymbol{Q b} \boldsymbol{b}_{n} \times \boldsymbol{d}_{n}
\end{array}\right]
$$

where $\times$ denotes the cross product. $\boldsymbol{W}$ depends on the cable robot geometry (points $A_{i}$ and $B_{i}$ ) and on the mobile platform pose $(\boldsymbol{Q}$ and $\boldsymbol{p})$. In static equilibrium, $\boldsymbol{f}+\boldsymbol{f}_{e}=\boldsymbol{0}$ where $f_{e}$ is the external wrench applied on the CDPR mobile platform.

\section{WRENCH FEASIBILITY ANALYSIS}

A mobile platform pose is said to be wrench-feasible [29], [30], when a required wrench set $R W$ is entirely contained within the set of wrenches $A W$ that the cables can apply on the mobile platform:

$$
R W \subseteq A W
$$

where $A W$ is called the available wrench set.

The $R W$ is the set of wrenches $\boldsymbol{f}$ that the cables must generate at the mobile platform reference point $P$ and its exact definition depends on the task(s) to be accomplished by the CDPR. The RW can be defined as the set of wrenches that permits to balance the total weight of the mobile platform and the payload, possibly for a set of payload masses and center of mass (CoM) positions [2].

The $A W$ is generally defined from admissible minimum and maximum cable tension values:

$$
\boldsymbol{t}_{\min } \leq \boldsymbol{t} \leq \boldsymbol{t}_{\max }
$$

where $\boldsymbol{t}_{\min } \geq \boldsymbol{0}$ is the vector of minimum tensions and $\boldsymbol{t}_{\max }$ is the vector of maximum tensions. The components $t_{\min }$ of $\boldsymbol{t}_{\min }$ must be non-negative since the cables cannot push on the mobile platform. A maximum tension $t_{\max }$ is defined to account for the capabilities and limitations of the cables, motors, supports, etc. In this paper, the maximum cable tension $t_{\max }$ is taken as the parameter to be optimized in the geometry optimization procedure. Note that the minimum and maximum cable tensions may be defined differently for each cable.

The bounds on the cable tensions in (4) forms an hypercube in the cable tension space. Being the image of this hypercube under the linear map represented by matrix $\boldsymbol{W}$, the $A W$ is a convex polytope which can be represented as the solution set of a system of linear inequalities in the following form [31]:

$$
A W=\{\boldsymbol{f} \mid \boldsymbol{C} \boldsymbol{f} \leq \boldsymbol{d}\}
$$

where matrix $\boldsymbol{C}$ and vector $\boldsymbol{d}$ can be obtained by means of the hyperplane shifting method introduced in [30].

An advantage of the formulation in (5) is that the wrenchfeasibility condition (3) can be tested by verifying if all wrenches in the $R W$ satisfy all the linear inequalities defined in (5). For simple definitions of the $R W$, the corresponding calculations are generally straightforward. In the next section, the representation of the $A W$ in (5) is used to obtain an expression of the maximum cable tension $t_{\max }$.
According to [30], each combination of five linearly independent columns $\boldsymbol{w}_{i_{1}}, \boldsymbol{w}_{i_{2}}, \boldsymbol{w}_{i_{3}}, \boldsymbol{w}_{i_{4}}$, and $\boldsymbol{w}_{i_{5}}$, of $\boldsymbol{W}$ provides two rows of $\boldsymbol{C}$, say $\boldsymbol{c}_{k}$ and $\boldsymbol{c}_{l}$. The row vectors $\boldsymbol{c}_{k}$ and $\boldsymbol{c}_{l}$ span the nullspace of matrix $\boldsymbol{M}_{I}=\left[\boldsymbol{w}_{i_{1}}, \boldsymbol{w}_{i_{2}}, \boldsymbol{w}_{i_{3}}, \boldsymbol{w}_{i_{4}}, \boldsymbol{w}_{i_{5}}\right]^{T}$ and are opposite $\left(\boldsymbol{c}_{k}=-\boldsymbol{c}_{l}=\operatorname{null}\left(\boldsymbol{M}_{I}\right)\right)$, where $I$ denotes the index set $\left\{i_{1}, \ldots, i_{5}\right\} \subset\{1, \ldots, n\}$. The element $d_{j}$ of $\boldsymbol{d}$ corresponding to the row $\boldsymbol{c}_{j}$ of $\boldsymbol{C}$ is given by:

$$
d_{j}=\sum_{i \in I_{j}^{+}} t_{\max } \boldsymbol{c}_{j} \boldsymbol{w}_{i}+\sum_{i \in I_{j}^{-}} t_{\min } \boldsymbol{c}_{j} \boldsymbol{w}_{i}
$$

where $I_{j}^{+}$and $I_{j}^{-}$are the subsets of $\{1, \ldots, n\}$ defined as $I_{j}^{+}=\left\{i \mid \boldsymbol{c}_{j} \boldsymbol{w}_{i}>0\right\}$ and $I_{j}^{-}=\left\{i \mid \boldsymbol{c}_{j} \boldsymbol{w}_{i}<0\right\}$.

\section{Maximum Tension Calculation}

First, let us consider that all cables have the same maximum cable tension $t_{\max }$ (and the same $t_{\text {min }}$ ). Let us also consider a given pose of the mobile platform (i.e. a given wrench matrix $\boldsymbol{W}$ ) and a given wrench $\boldsymbol{f}$. Substituting the expression (6) of $d_{j}$ in the system of linear inequalities $\boldsymbol{C} \boldsymbol{f} \leq \boldsymbol{d}$ of (5) and considering the $j^{\text {th }}$ row of this system, the following inequality is obtained:

$$
t_{\max } \geq \frac{\boldsymbol{c}_{j} \boldsymbol{f}-t_{\min } \sum_{i \in I_{j}^{-}} \boldsymbol{c}_{j} \boldsymbol{w}_{i}}{\sum_{i \in I_{j}^{+}} \boldsymbol{c}_{j} \boldsymbol{w}_{i}}
$$

In the particular case $I_{j}^{+}=\emptyset$, the denominator in (7) is equal to zero, $t_{\max }$ is not involved in the $j^{\text {th }}$ row of $\boldsymbol{C} \boldsymbol{f} \leq \boldsymbol{d}$, and the following condition on $t_{\min }$ must be satisfied:

$$
\boldsymbol{c}_{j} \boldsymbol{f} \leq t_{\min } \sum_{i=1}^{n} \boldsymbol{c}_{j} \boldsymbol{w}_{i}
$$

The maximum cable tension $t_{\max }$ must verify (7) for all inequalities in $\boldsymbol{C} \boldsymbol{f} \leq \boldsymbol{d}$, i.e.:

$$
t_{\max } \geq \max _{j} \frac{\boldsymbol{c}_{j} \boldsymbol{f}-t_{\min } \sum_{i \in I_{j}^{-}} \boldsymbol{c}_{j} \boldsymbol{w}_{i}}{\sum_{i \in I_{j}^{+}} \boldsymbol{c}_{j} \boldsymbol{w}_{i}}
$$

For a given pose of the mobile platform and a given $t_{m i n}$, the right-hand side of (9) gives the lowest value of $t_{\text {max }}$ such that a given wrench $\boldsymbol{f}$ is feasible, i.e., can be generated at the mobile platform with cable tensions $t$ verifying (4).

In order to determine the lowest value of $t_{\max }$ such a $R W$ is feasible, i.e. such that (3) is verified, a particular definition of $R W$ should be considered.

In this paper, the CDPR is intended to work on the facade of a building, operating in a quasi-static manner while carrying heavy payloads. Consequently, a first definition of interest for the RW is the one presented in [2]. This RW is defined as the set of wrenches $\boldsymbol{f}=\left[f_{x}, f_{y}, f_{z}, \tau_{x}, \tau_{y}, \tau_{z}\right]^{T}$ allowing to balance a total (platform and payload) mass $m$, $m_{\min } \leq m \leq m_{\max }$, where the projection of the $C o M$ on the horizontal plane containing the platform reference point $P$ can be located anywhere in a disc of radius $r$ centered at $P$ : 


$$
R W_{\text {circular }}=\left\{\begin{array}{c}
\boldsymbol{f} \mid f_{x}=f_{y}=\tau_{z}=0, \\
m_{\min } g \leq f_{z} \leq m_{\max } g \\
\sqrt{\tau_{x}^{2}+\tau_{y}^{2}} \leq f_{z} r
\end{array}\right\}
$$

where $g$ is the gravity acceleration.

Another definition of interest for the $R W$ is similar to the previous one but with the projection of the $C o M$ located in a square horizontal zone of dimensions $2 s \times 2 s$ ( $s$ denotes the half-side length of the square) along the $x$ and $y$ directions ( $z$ being the vertical axis as shown in Fig. 1) :

$$
R W_{\text {square }}=\left\{\begin{array}{c}
\boldsymbol{f} \mid f_{x}=f_{y}=\tau_{z}=0, \\
m_{\min } g \leq f_{z} \leq m_{\max } g, \\
\left|\tau_{x}\right| \leq f_{z} s,\left|\tau_{y}\right| \leq f_{z} s
\end{array}\right\}
$$

In this second definition of the $R W$, the CoM can be shifted to the extrema along $x$ and $y$ simultaneously. The square and circular zones where the CoM can be located are illustrated in Fig. 2.
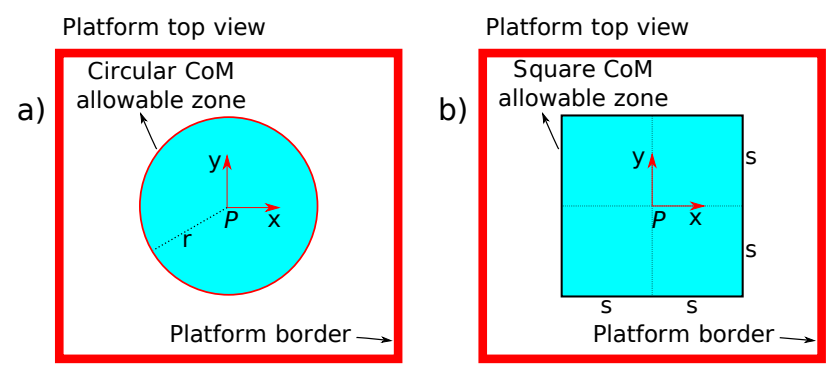

Fig. 2. Circular and square horizontal CoM allowable zones.

In order to determine the lowest value of $t_{\max }$ such that $R W_{\text {circular }}$ is feasible, note that for each row $\boldsymbol{c}_{j}$ of $\boldsymbol{C}$, the wrench $\boldsymbol{f} \in R W_{\text {circular }}$ which maximizes $\boldsymbol{c}_{j} \boldsymbol{f}$ is given by:

$$
\begin{gathered}
f_{x}=f_{y}=\tau_{z}=0, \\
f_{z}=\left\{\begin{array}{c}
m_{\max } g \text { if } c_{j f z} \geq-r \sqrt{c_{j \tau x}^{2}+c_{j \tau y}^{2}} \\
m_{\min } g \text { if } c_{j f z}<-r \sqrt{c_{j \tau x}^{2}+c_{j \tau y}^{2}}
\end{array}\right. \\
\tau_{x}=f_{z} r \frac{c_{j \tau x}}{\sqrt{c_{j \tau x}^{2}+c_{j \tau y}^{2}}}, \tau_{y}=f_{z} r \frac{c_{j \tau y}}{\sqrt{c_{j \tau x}^{2}+c_{j \tau y}^{2}}}
\end{gathered}
$$

where $\boldsymbol{c}_{j}=\left[c_{j f x}, c_{j f y}, c_{j f z}, c_{j \tau x}, c_{j \tau y}, c_{j \tau z}\right]$.

In the case of the square horizontal CoM allowable zone, the wrench $\boldsymbol{f} \in R W_{\text {square }}$ which maximizes $\boldsymbol{c}_{j} \boldsymbol{f}$ is given by:

$$
\begin{aligned}
& f_{x}=f_{y}=\tau_{z}=0, \\
& f_{z}= \begin{cases}m_{\max } g & \text { if } c_{j f z} \geq-s\left(\left|c_{j \tau x}\right|+\left|c_{j \tau y}\right|\right) \\
m_{\min } g & \text { if } c_{j f z}<-s\left(\left|c_{j \tau x}\right|+\left|c_{j \tau y}\right|\right)\end{cases} \\
& \tau_{x}=f_{z} s \cdot \operatorname{sgn}\left(c_{j \tau x}\right), \tau_{y}=f_{z} s \cdot \operatorname{sgn}\left(c_{j \tau y}\right)
\end{aligned}
$$

where the sign function is defined as $\operatorname{sgn}(x)=1$ if $x \geq 0$ and $\operatorname{sgn}(x)=-1$ if $x<0$.

Let $\boldsymbol{f}_{j}$ be the wrench defined by (12) in the case of $R W_{\text {circular }}$ and by (13) in the case of $R W_{\text {square }}$ Then, according to (9), the lowest value of $t_{\max }$ such that $R W_{\text {circular }}$ or $R W_{\text {square }}$ is feasible is equal to:

$$
t_{\max }=\max _{j} \frac{\boldsymbol{c}_{j} \boldsymbol{f}_{j}-t_{\min } \sum_{i \in I_{j}^{-}} \boldsymbol{c}_{j} \boldsymbol{w}_{i}}{\sum_{i \in I_{j}^{+}} \boldsymbol{c}_{j} \boldsymbol{w}_{i}}
$$

The advantage of (14) is to give an explicit expression of the lowest value of $t_{\max }$ such that $R W_{\text {circular }}$ or $R W_{\text {square }}$ is feasible. Otherwise, i.e. without the formulation in (14), a sweeping of the wrenches over the $R W$ is necessary to determine the lowest value of $t_{\max }$.

In (14), the maximum cable tensions are assumed to be the same for all the cables. However, one specificity of fullyconstrained cable robots operating on a building facade is that the cables connected to the roof supports must withstand the totality of the payload in addition to the load due to the bottom cable tensions. In practice, the cable tensions are thus larger in the top (upper) cables than in the bottom (lower) cables.

Hence, a further refinement of the maximum cable tension evaluation is to consider different values of the maximum cable tensions between lower and upper cables $\left(t_{\operatorname{maxl}}\right.$ and $t_{\text {maxh }}$, respectively), while conserving the same value of $t_{\max }$ obtained previously in (14) for the top cables $\left(t_{\operatorname{maxh}}=\right.$ $\left.t_{\max }\right)$.

The value of $t_{\operatorname{maxl}}$ is calculated from the wrench feasibility condition $(\boldsymbol{C} \boldsymbol{f} \leq \boldsymbol{d})$ by separating the components of $\boldsymbol{t}_{\max }$ in two sets, one related to the lower cables and the other one to the upper cables. The lowest value of $t_{\operatorname{maxl}}$ is then equal to:

$$
t_{\text {max }}=\max _{j} \frac{\boldsymbol{c}_{j} \boldsymbol{f}_{j}-t_{\min } \sum_{i \in I_{j}^{-}} \boldsymbol{c}_{j} \boldsymbol{w}_{i}-t_{\operatorname{maxh}} \sum_{i \in I_{j h}^{+}} \boldsymbol{c}_{j} \boldsymbol{w}_{i}}{\sum_{i \in I_{j l}^{+}} \boldsymbol{c}_{j} \boldsymbol{w}_{i}}
$$

where $I_{j h}^{+}$and $I_{j l}^{+}$are the subsets of $I_{j}^{+}$corresponding to the upper and lower cable indices, respectively. Note that, if $I_{j l}^{+}$ is empty, the corresponding condition on $t_{\operatorname{maxl}}$ is neglected.

The expressions of the lowest maximum cable tensions obtained in (14) and (15) have been derived for a given pose of the CDPR mobile platform (i.e., for a given wrench matrix $\boldsymbol{W}$ ). Hence, the expressions in (14) and (15) must be calculated over the prescribed workspace of the CDPR. The maximum value obtained in these calculations is the lowest maximum cable tension such that (3) is verified across the CDPR workspace.

\section{Workspace AND BASE AND PlatForm Geometries}

Input variables to the geometry optimization problem are discussed in the present section. First of all, the workspace is defined in Section V-A. In this paper, the geometry optimization is based on the consideration of a finite number of platform poses distributed across the prescribed workspace. These poses are defined in this section. Moreover, the parametrization of the possible platform and base geometries are presented in Section V-B. 

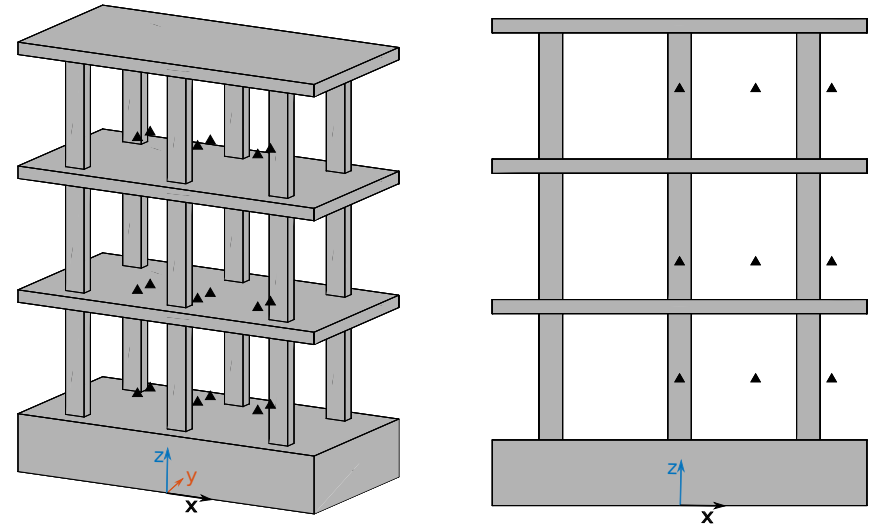

Fig. 3. Workspace positions from perspective and front views.

\section{A. Workspace definition}

The studied CDPR is meant to be used during the construction or maintenance of building facades. The platform should be able to move in front of the whole facade of the building. Therefore, the workspace over $x z$ is the surface of the facade where the $x$ and $z$ axes are shown in Fig. 3. For the considered example building, this surface is a rectangle of height $10.2 \mathrm{~m}$ and width $8.5 \mathrm{~m}$. The depth along the $y$ axis has also to be defined. The platform should be able to move close enough to the facade. The minimum distance between the border of the platform and the building facade is set to $40 \mathrm{~cm}$ in the example dealt with in this paper. Moreover, a safe distance of $1 \mathrm{~m}$ along the $y$ axis is required during the movement of the platform in front of the building. It should be highlighted that these dimensions were defined taking into account the border of the platform. Consequently, the positions of the platform reference point $P$ with respect to the building depends on the platform dimensions.

The performance of the CDPR will be evaluated at a finite number of positions in the workspace described above. Both the building and the CDPR are considered to be symmetric with respect to the $y z$ plane. Consequently, the analysis of half of the workspace is sufficient. In the $x$ direction, the positions are distributed as shown in Fig. 3: Three at the middle of the building, three on its edge, and three others at the middle between them. In the $y$ direction, the positions are placed at the minimal distance with respect to the building facade $(40 \mathrm{~cm})$ and at the safe moving distance $(1 \mathrm{~m})$. Moreover, small platform orientation angles about the $\mathrm{z}$ axis, distributed between $-2^{\circ}$ and $2^{\circ}$, are considered at each position defined above. These angle limitations are small because they are only set to handle possible construction deviations along the building facade.

\section{B. CDPR base and platform geometries}

The geometric design of a CDPR includes the determination of the positions of the cable drawing points at the base, of the attachment points on the platform, and of cable connections between these two sets of points. Considering the shape of the workspace discussed in the previous section,
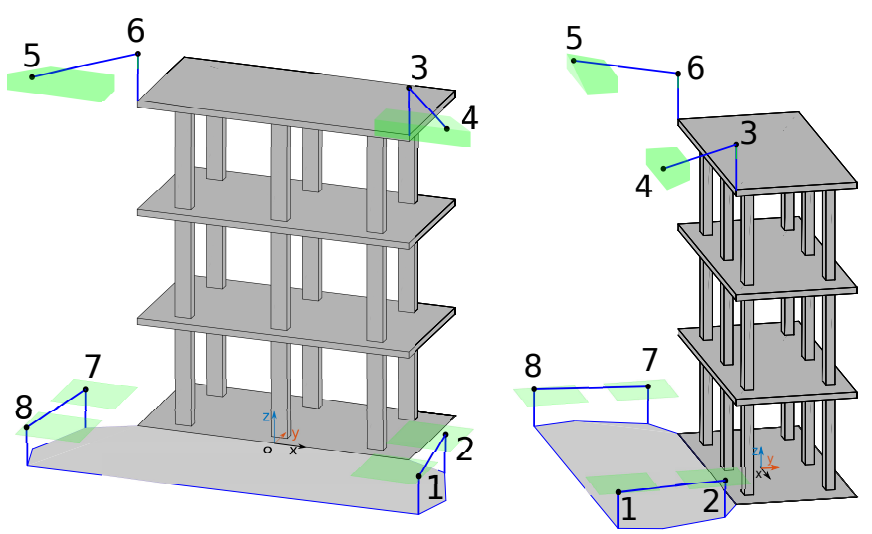

Fig. 4. Base geometry with 8 cables (same configuration shown from two different angles).

8 cables turns out to be an appropriate choice for the fullyconstrained CDPR considered in this paper. The attachment points on the CDPR platform are defined as the 8 points of a rectangular parallelepiped. Besides, four cables drawing points are positioned at the top of the building and the remaining four are positioned at the ground level (points 1, 2, 7 and 8). These base and platform geometries are depicted in Fig. 4 and Fig. 5, respectively. Considering symmetry with respect to the middle $y z$ plane, the drawing points numbered $n, n-1, \ldots, 2,1$ are symmetric to the drawing points numbered $1,2, \ldots, n-1, n$, respectively. Thereby, by symmetry, only 4 drawing point positions have to be optimized for $n=8$.

The green areas in Fig. 4 represent the zones where the drawing points can be located. These areas are defined to cover the prescribed workspace by the drawing points while reducing as much as possible the extension outside of the building. A maximum distance of $2 m$ to the sides (in the $x$ direction) and up to $5 \mathrm{~m}$ in front of the building facade (in the negative $y$ direction) are available for the drawing points at the ground level. The height of these drawing points (1, 2, 7 and 8 ) is set to $1.2 \mathrm{~m}$ to avoid collisions with some stacks of objects which may clutter the ground level. The remaining 4 drawing points are positioned at the top of the building. More precisely, they are located above the building at a height of $1 \mathrm{~m}$ up to $1.5 \mathrm{~m}$. The drawing points 4 and 5 are shifted outwards the facade in an effort to enlarge the CDPR workspace.

The CDPR mobile platform dimensions are more related to the facade elements to be installed and to the size of components placed on-board the platform. The platform geometry parametrization is shown in Fig. 5. It consists of three geometric parameters whose (range of possible) values are indicated in Table I.

TABLE I

GEOMETRIC PARAMETERS OF THE MOBILE PLATFORM

\begin{tabular}{|c|c|c|}
\hline$w_{p}$ & $t_{p}$ & $h_{p}$ \\
\hline 1.5 & {$[1,1.5]$} & {$[2,3]$} \\
\hline
\end{tabular}

A CDPR cable configuration is completed with the defini- 


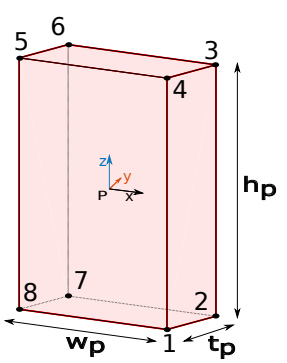

Fig. 5. Platform geometry with 8 cables.

tion of the cable connections between the drawing points (base geometry) and the attachment points (platform geometry). In order to consider in the geometry optimization problem all possible cable connections between the base and platform geometries defined above, a combinatorial problem must be dealt with. A unique pair of platform and base geometry with $n$ cables connected between them leads to $C_{n}^{n^{2}}$ possibilities (4.4 $10^{9}$ possibilities for 8 cables). Nevertheless, some constraints can be defined so as to decrease this number of combinations to a more reasonable number. As discussed earlier, in this paper, the CDPR is symmetric with respect to the $y z$ plane. Cable connections violating this constraint are disregarded. In addition, collisions between cables and the platform are predicted.

\section{DESIGN CRITERIA}

In order to proceed with the geometry optimization, the objective function and the constraints should be defined. The ones used in the present study are presented in this section.

\section{A. Cable tension reduction}

The CDPR cost is partly related to the maximum cable tensions. In fact, the smallest possible maximum cable tension is sought. Accordingly, the lowest maximum cable tensions calculated in (14) (and (15)) are defined as the objective function to be minimized. As briefly discussed at the end of Section IV, the lowest maximum cable tension should be computed at various poses across the CDPR workspace. In this paper, the finite number of workspace poses described in Section V-A are considered. The maximal value among these poses is considered as the performance index for a given CDPR geometry.

\section{B. Cable collisions}

Another criterion that must be considered in the design of the CDPR is the avoidance of collisions between the cables and between the cables and the mobile platform, across the CDPR workspace. For instance, a collision can be tested using the distance between cables over all the workspace poses. This distance must be larger than the cable diameters. Cable collisions are tested at the finite number of workspace poses described in Section V-A while the absence of cable collisions across the workspace is verified afterward the CDPR geometry optimization procedure. Additionally, the cables must not pass throughout the mobile platform. This condition is mainly considered in the generation of the cable configurations between drawing points and attachment points of the CDPR.

\section{GEOMETRIC DESIGN STUDY}

The geometric design study in this paper aims to select and optimize the CDPR geometry based on the performance index defined in Section VI-A. Following the methodology used in [2], the design study is based on two phases. The first phase consists in testing a very large number of possible cable connections between various base and platform preselected geometries. The second phase aims at refining the result of the first phase using an optimization algorithm. The design methodology in the two phases and the design results are presented in this section.

\section{A. Phase 1: Cable configurations}

In this first phase, the workspace poses considered are those defined in Section V-A, and the base and platform geometry parametrizations are defined in Section V-B. All the possible cable connections between the corresponding base and platform points are first generated according to Section V-B. All of the CDPR geometries thereby obtained are then tested in order to retain the best ones. A geometry without cable collisions is considered better than another one according to the objective function presented in Section VIA.

\section{B. Phase 2: Geometric parameter optimization}

The first phase is followed by a second phase in order to refine the dimensions of the CDPR. This second phase is based on a sequential quadratic programming optimization. Referring to Section VI-A, the optimization problem in this paper consists in minimizing the maximum cable tension $t_{\max }$ while variating the geometrical dimensions of the drawing points $\left(\boldsymbol{x}_{d}\right)$ and attachment points $\left(\boldsymbol{x}_{a}\right)$ :

$$
\begin{array}{cc}
\underset{\boldsymbol{x}_{d}, \boldsymbol{x}_{a}}{\operatorname{minimize}} & t_{\max } \\
\text { subject to } & \left\{\begin{array}{c}
t_{\max }=t_{\text {max }}^{\boldsymbol{x}_{d}, \boldsymbol{x}_{a}} \\
\text { avoid collision }
\end{array}\right.
\end{array}
$$

where $t_{\max }^{\boldsymbol{x}_{d}, \boldsymbol{x}_{a}}$ is the maximum of $t_{\max }$ (evaluated using (14)) over the workspace poses (defined in section V-A). For each pose, $t_{\max }$ is calculated by considering the geometrical parameters $\boldsymbol{x}_{d}$ and $\boldsymbol{x}_{a}$ defining the drawing and attachment points dimensions.

Various other design constraints may be defined according to application requirements such as improving the stiffness, avoid collision with an obstacle, etc. The best CDPR geometry found in the first phase is considered as the initial guess for the optimization problem of the second phase. Note that in this second phase, only the geometric dimensions are optimized while the cable connections remain the same as the one obtained in the first phase.

After the second phase, the best cable robot geometry and the corresponding value of the lowest $t_{\max }$ are obtained. However, the maximum cable tensions of the lower cables $t_{\operatorname{maxl}}$ can be further reduced as explained at the end of Section IV. The lowest value of $t_{\operatorname{maxl}}$ for the optimal geometry is evaluated using (15). 

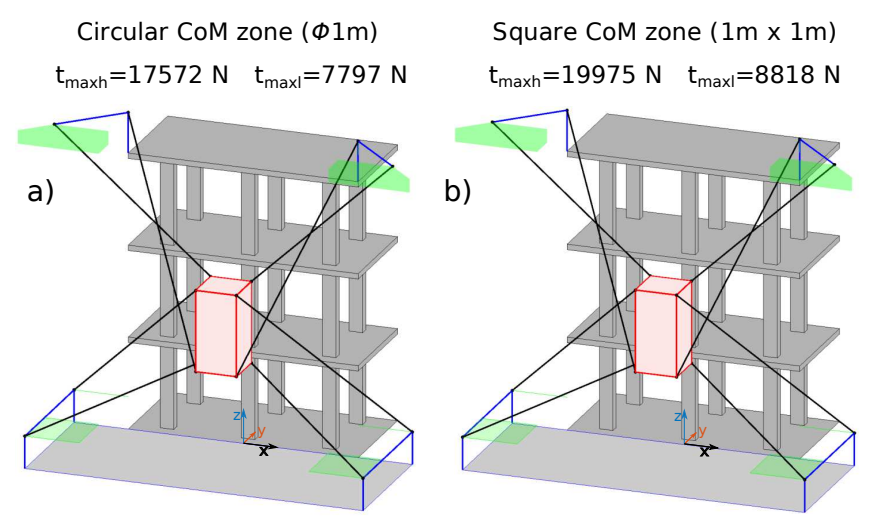

Fig. 6. Best CDPR geometries obtained after phases 1 and 2 of the design with consideration of circular $\left(R W_{\text {circular }}\right.$, subfigure a) and square $\left(R W_{\text {square }}\right.$, subfigure b) CoM allowable zones.

\section{Results and discussion}

In the context of the EU project Hephaestus, a prototype of the CDPR whose geometry optimization is the subject of this paper should be installed on a demonstration building facade. A computer code has been developed to select and optimize this CDPR geometry according to the methodology presented in the previous sections. Running this code for the CDPR possible geometries and the optimization problem defined in this paper, for a maximum mass $m_{\max }=1000$ $\mathrm{kg}$, a minimum mass $m_{\min }=200 \mathrm{~kg}$, a minimum cable tension $t_{\min }=200 \mathrm{~N}$ and the two definitions of the required wrench set $R W_{\text {square }}$ and $R W_{\text {circular }}$ given in Section IV with $r=s=0.5 \mathrm{~m}$, the best CDPR geometry obtained after the second phase for the two $R W$ cases is shown in Fig. 6. Several hours of computation time (on a standard computer, Intel Core i7- 7820HQ @ 2.9 GHZ 16 GB RAM) were needed to obtain this result.

This best CDPR geometry has been obtained in both the case of $R W_{\text {square }}$ and $R W_{\text {circular. }}$. However, the values of the lowest maximum cable tensions $\left(t_{\operatorname{maxh}}\right.$ and $\left.t_{\operatorname{maxl}}\right)$ are larger in the case of $R W_{\text {square }}$ which can be expected since $r=s=0.5 \mathrm{~m}$ and, thus, all wrenches in $R W_{\text {circular }}$ are contained in $R W_{\text {square }}$.

It is worth noting that the CDPR geometry obtained in this paper (Fig. 6) possesses smaller lowest maximum cable tensions than CDPR geometries similar to the ones used in existing fully-constrained 8-cable CDPRs such as IPAnema [7], CaBLAR [9], Media-TIC [32] and CableEndy, as illustrated in Fig. 7. In this figure, the best values of the lowest maximum cable tensions are indicated. These values have been obtained using the optimization problem defined in this paper (second phase, Section VII-B), where the way of connecting the cables between the CDPR base and the platform is similar to the one of the aforementioned fullyconstrained 8-cable CDPRs.

With the proposed method where the lowest maximum cable tensions is minimized, it is possible to optimize CDPR geometries having more than eight cables, which should lead to a smaller values of the maximum cable tensions. The corresponding optimal CDPR geometries are not shown in

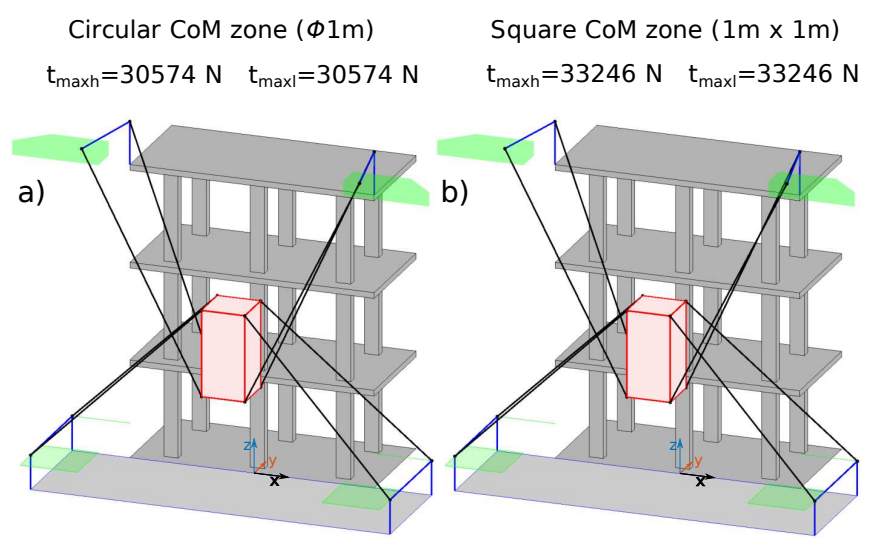

Fig. 7. Best CDPR geometries obtained for predefined cable connections with consideration of circular $\left(R W_{\text {circular }}\right.$, subfigure a) and square ( $R W_{\text {square }}$, subfigure b) CoM allowable zones.

this paper for short. However, a choice have to be made between having more cables with lower maximum cable tensions, or less cables with larger maximum cable tensions. The selection of the number of cables is thus a trade-off between, on the one hand, the number of CDPR components (winches, drums, pulleys, supports, etc.) and, on the other hand, the size, capability, and cost of these components.

In the specific application of the CDPR in the Hephaestus project, it is required to handle heavy payloads that will be located on the mobile platform between the platform and the building. This implies a shift of the zone of possible CoM locations towards the building facade. Future works will focus on the definition of such CoM allowable zones, of the corresponding $R W$, and on the calculation of the lowest $t_{\max }$ for this $R W$. Moreover, in construction environments, lateral disturbances due to winds, as well as contacts or collisions with an object can probably occur. To make the CDPR robust to such disturbances, a minimum stiffness should be targeted in the design. Therefore, another perspective of the current work is to include constraints on the CDPR stiffness in the CDPR geometry optimization. A first approach may address the stiffness in a specific direction. Similarly to [33], the stiffness in the $y$ direction is critical to the present application. A lower bound for this directional stiffness may thus be imposed as an additional constraint. Otherwise, a more complex approach may analyze the displacement of a given point of the platform according to a homogenized stiffness matrix, as discussed in [34]. Finally, the geometric tolerances on the cable drawing points is normally high in construction sites. The determination of $t_{\max }$ while considering these tolerances may also be part of future works.

\section{CONCLUSION}

The optimization of the geometry of a large CDPR intended to work on building facades was dealt with in this paper. An explicit formulation of the lowest maximum cable tension that ensures the validity of wrench-feasibility constraints was first proposed. This lowest maximum cable tension formulation was used as the objective function of 
the CDPR geometry optimization problem since the maximum cable tension is a critical parameter in the design of the components of a CDPR. Applying this geometry optimization to an example case of a large CDPR intended to work on a building facade, an original fully-constrained 8cable CDPR geometry was obtained and compared in term of maximum cable tension to an existing fully-constrained CDPR geometry.

\section{ACKNOWLEDGMENT}

The research leading to these results has received funding from the European Union's H2020 Programme (H2020/2014-2020) under grant agreement No. 732513.

\section{REFERENCES}

[1] J. Albus, R. Bostelman, and N. Dagalakis, "The NIST robocrane," Journal of Field Robotics, vol. 10, no. 5, pp. 709-724, 1993.

[2] M. Gouttefarde, J.-F. Collard, N. Riehl, and C. Baradat, "Geometry selection of a redundantly actuated cable-suspended parallel robot," IEEE Transactions on Robotics, vol. 31, no. 2, pp. 501-510, 2015.

[3] R. R. Thompson and M. S. Blackstone, "Three-dimensional moving camera assembly with an informational cover housing," Mar. 29 2005, US Patent 6,873,355.

[4] S. Kawamura, H. Kino, and C. Won, "High-speed manipulation by using parallel wire-driven robots," Robotica, vol. 18, pp. 13-21, 2000.

[5] M. Gouttefarde and C. M. Gosselin, "Analysis of the wrench-closure workspace of planar parallel cable-driven mechanisms," IEEE Transactions on Robotics, vol. 22, no. 3, pp. 434-445, 2006.

[6] A. Pott, H. Mütherich, W. Kraus, V. Schmidt, P. Miermeister, and A. Verl, "IPAnema: a family of cable-driven parallel robots for industrial applications," in Cable-Driven Parallel Robots. Springer, 2013, pp. 119-134.

[7] W. Kraus, A. Spiller, and A. Pott, "Energy efficiency of cable-driven parallel robots," in Robotics and Automation (ICRA), 2016 IEEE International Conference on. IEEE, 2016, pp. 894-901.

[8] P. Lafourcade, M. Llibre, and C. Reboulet, "Design of a parallel wiredriven manipulator for wind tunnels," in Proceedings of the Workshop on Fundamental Issues and Future Research Directions for Parallel Mechanisms and Manipulators. Quebec City, Canada, 2002, pp. 187194.

[9] T. Bruckmann, W. Lalo, K. Nguyen, and B. Salah, "Development of a storage retrieval machine for high racks using a wire robot," in ASME 2012 International Design Engineering Technical Conferences and Computers and Information in Engineering Conference, 2012, pp. 771-780.

[10] L. Gagliardini, S. Caro, M. Gouttefarde, P. Wenger, and A. Girin, "Optimal design of cable-driven parallel robots for large industrial structures," in Robotics and Automation (ICRA), 2014 IEEE International Conference on. IEEE, 2014, pp. 5744-5749.

[11] T. Bock, "Construction robotics enabling innovative disruption and social supportability," in ISARC. Proceedings of the International Symposium on Automation and Robotics in Construction, vol. 32. Vilnius Gediminas Technical University, Department of Construction Economics \& Property, 2015.

[12] S. Tadokoro, S. Nishioka, T. Kimura, M. Hattori, T. Takamori, and K. Maeda, "On fundamental design of wire configurations of wiredriven parallel manipulators with redundancy," in ASME Japan/USA symposium on flexible automotion, vol. 1, 1996, pp. 151-158.

[13] J. Pusey, A. Fattah, S. Agrawal, and E. Messina, "Design and workspace analysis of a 6-6 cable-suspended parallel robot," Mechanism and machine theory, vol. 39, no. 7, pp. 761-778, 2004.

[14] M. Hiller, S. Fang, S. Mielczarek, R. Verhoeven, and D. Franitza, "Design, analysis and realization of tendon-based parallel manipulators," Mechanism and Machine Theory, vol. 40, no. 4, pp. 429-445, 2005.

[15] S. Perreault and C. M. Gosselin, "Cable-driven parallel mechanisms: Application to a locomotion interface," ASME J. Mech. Des., vol. 130, no. 10 , Oct. 2008.

[16] M. M. Aref, H. D. Taghirad, and S. Barissi, "Optimal design of dexterous cable driven parallel manipulators," International Journal of Robotics, 2009.
[17] G. Rosati, D. Zanotto, and S. K. Agrawal, "On the Design of Adaptive Cable-Driven Systems," Journal of Mechanisms and Robotics, vol. 3, no. 2,2011

[18] M. Gouttefarde, D. Daney, and J.-P. Merlet, "Interval-analysis-based determination of the wrench-feasible workspace of parallel cabledriven robots," IEEE Transactions on Robotics, vol. 27, no. 1, pp. $1-13,2011$

[19] G. Abbasnejad, J. Yoon, and H. Lee, "Optimum kinematic design of a planar cable-driven parallel robot with wrench-closure gait trajectory," Mechanism and Machine Theory, vol. 99, pp. 1-18, 2016.

[20] H. Lamine, S. Bennour, and L. Romdhane, "Design of cable-driven parallel manipulators for a specific workspace using interval analysis," Advanced Robotics, vol. 30, no. 9, pp. 585-594, 2016.

[21] F. Guay, P. Cardou, A. L. Cruz-Ruiz, and S. Caro, "Measuring how well a structure supports varying external wrenches," in Mechanisms and Machine Science. Springer, 2014, vol. 17, pp. 385-392.

[22] S. Behzadipour and A. Khajepour, "Stiffness of cable-based parallel manipulators with application to stability analysis," Journal of Mechanical Design, vol. 128, no. 1, 2006.

[23] S. Abdolshah, D. Zanotto, G. Rosati, and S. K. Agrawal, "Optimizing stiffness and dexterity of planar adaptive cable-driven parallel robots," Journal of Mechanisms and Robotics, vol. 9, no. 3, p. 31004, 2017.

[24] D. Zanotto, G. Rosati, and A. Rossi, "Performance analysis of planar cable-based parallel manipulators," in ASME 2010 10th Biennial Conference on Engineering Systems Design and Analysis, 2010, pp. 789-798.

[25] H. R. Fahham and M. Farid, "Optimum design of planar redundant cable-suspended robots for minimum time trajectory tracking," in Control Automation and Systems (ICCAS), 2010 International Conference on. IEEE, 2010, pp. 2156-2163.

[26] J. T. Bryson, X. Jin, and S. K. Agrawal, "Configuration robustness analysis of the optimal design of cable-driven manipulators," Journal of Mechanisms and Robotics, vol. 8, no. 6, 2016.

[27] — "Optimal Design of Cable-Driven Manipulators Using Particle Swarm Optimization," Journal of Mechanisms and Robotics, vol. 8, no. 4,2016

[28] T. Bruckmann, L. Mikelsons, and M. Hiller, "A design-to-task approach for wire robots," in Interdisciplinary Applications of Kinematics. Springer, 2012, pp. 83-97.

[29] P. Bosscher, A. T. Riechel, and I. Ebert-Uphoff, "Wrench-feasible workspace generation for cable-driven robots," IEEE Transactions on Robotics, vol. 22, no. 5, pp. 890-902, 2006.

[30] S. Bouchard, C. Gosselin, and B. Moore, "On the ability of a cabledriven robot to generate a prescribed set of wrenches," Journal of Mechanisms and Robotics, vol. 2, no. 1, 2010.

[31] G. M. Ziegler, Lectures on polytopes. Springer, 2012, vol. 152.

[32] J.-B. Izard, M. Gouttefarde, C. Baradat, D. Culla, and D. Sallé, "Integration of a parallel cable-driven robot on an existing building façade," in Cable-driven parallel robots. Springer, 2013, pp. 149164.

[33] H. Jamshidifar, A. Khajepour, B. Fidan, and M. Rushton, "Kinematically-Constrained Redundant Cable-Driven Parallel Robots: Modeling, Redundancy Analysis, and Stiffness Optimization," IEEE/ASME Transactions on Mechatronics, vol. 22, no. 2, pp. 921930, 2017.

[34] D. Q. Nguyen and M. Gouttefarde, "Stiffness Matrix of 6-DOF CableDriven Parallel Robots and Its Homogenization," in Advances in Robot Kinematics. Springer, 2014, pp. 181-191. 